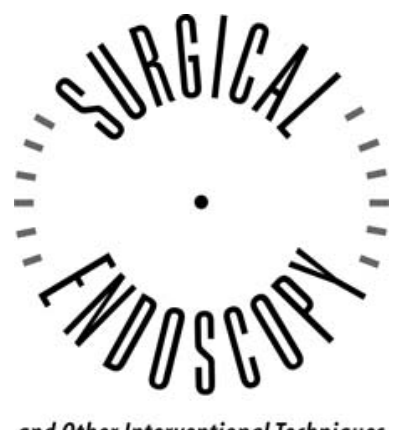

\title{
Friction dynamics of trocars
}

\author{
J. J. van den Dobbelsteen, A. Schooleman, J. Dankelman
}

Department of Biomechanical Engineering, Delft University of Technology, Mekelweg 2, NL-2628 CD, Delft, The Netherlands

Received: 4 May 2006/Accepted: 28 July 2006/Online publication: 13 December 2006

\begin{abstract}
Background: In minimally invasive surgery, force feedback information on tissue manipulation is altered by friction between the instrument and the sealing mechanism of the trocar. It is unknown how the different sealing mechanisms of currently available trocars influence the friction forces. The current study investigated the dynamic changes in friction for various trocars at different instrument velocities.

Methods: The friction characteristics for six common types of trocars were determined. A force sensor was attached to the shaft of a standard 5-mm disposable grasper to measure the forces required to move it through the trocars. Movement velocity and direction of the shaft were controlled by a servomotor. In addition, whether moistening the shaft reduced friction was tested.

Results: The friction depended on the type of trocar, the movement velocity, and the movement direction, and varied between 0.25 and $3.0 \mathrm{~N}$. Specifically, trocars with narrow sealing caps (i.e., high normal force onto the shaft) and trocars with thick sealing caps (i.e., large contact area) generate a high amount of friction. Moistening the shaft reduced friction $15 \%$ to $45 \%$. For most trocars, large fluctuations in forces occur when the movement starts or when the direction reverses. The magnitude of these fluctuations varied between 0.2 and $2.5 \mathrm{~N}$

Conclusions: For some trocars, friction can be as great as the forces associated with instrument-tissue interaction. At movement reversals, friction fluctuates due to deformations of the rubber and silicon parts of the sealing mechanism. Such high variance can deteriorate surgical performance during high precision tasks (e.g., tissue manipulation) that typically involve many changes in movement direction. Comparisons of the investigated trocars indicate that the friction magnitude and variance can be reduced easily by changing the properties of the sealing cap or by lubricating the instruments.
\end{abstract}

Correspondence to: J. J. van den Dobbelsteen
Key words: Force feedback - Friction - Laparoscopy - Tissue manipulation - Trocar

Accurate control of grasping and pulling forces is an essential component of many surgical activities. In tissue manipulation, inability to control these forces is associated with slippage of the tissue if the pinching forces are too low, and with damage to delicate tissue if the forces are too high $[1,6]$. The surgeon usually senses the magnitudes of applied forces peripherally via mechanoreceptors in the skin and muscles $[5,7]$. However, in minimally invasive surgery, the lack of direct manual contact between the tissue and the surgeon's fingers reduces the quality of force feedback on the surgeon's interactions with the tissue [2]. Pulling forces associated with instrument-tissue contact are masked by friction between the instrument and the sealing caps, and between the membranes and the valves of the trocars. This complicates the control of handling the tissue and of applying the exact force needed. As a result, the risk of tissue damage is increased.

A number of researchers have measured the frictional forces generated when surgical instruments rub against the inner parts of trocars $[3,8,10,11]$. Still, there is only limited knowledge about the dynamic nature of friction in trocars. To characterize the friction in trocars, Picod et al. [10, 11] used a friction model based on Coulomb friction and viscous friction. Such models are well established and show (nonlinear) increases in friction with increasing velocity. However, the assumption that friction force depends only on instantaneous velocity is in general not a good approximation [4]. At the start of a movement, adhesion of the surgical instrument, the sealing caps, and the valves of trocars will result in increasing forces until the molecular bonds break and a slip occurs. Also, at low velocities, the molecular bonds may continuously detach and attach so that the forces may oscillate with a high frequency [9].

The results of Picod et al. [10,11] suggest that especially for rapid surgical movements, friction will be detrimental for the utility of force feedback. Yet, the 
Table 1. Main characteristics of the different trocars

\begin{tabular}{lllll}
\hline Trocar & Sealing cap & Housing & Diameter ${ }^{\mathrm{b}}(\mathrm{mm})$ \\
\hline B Braun 5.5 & Rubber, 4 mm & Valve & Stainless steel nondisposable & 5.5 \\
Karl Storz 5 & Rubber, 4 mm & Silicon & Stainless steel spring-loaded & Stainless steel nondisposable \\
Endopath 12 & Silicon, 6 mm, ${ }^{\text {a }}$ additional reducer, 3 mm & Plastic spring-loaded & Plastic disposable & 12 \\
Endopath 5.5 & Silicon, 4 mm & Plastic spring-loaded & Plastic disposable & 5.5 \\
Xcel 12 & Rubber, plastic self-adjusting reducer lubricated & Rubber & Plastic disposable & 12 \\
Xcel 5 & None & Rubber & Plastic disposable & 5 \\
\hline
\end{tabular}

a Inner diameter of the sealing cap

${ }^{b}$ Maximal diameter of the shaft of the instrument for which the trocar is suitable

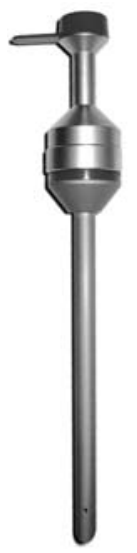

B Braun 5.5

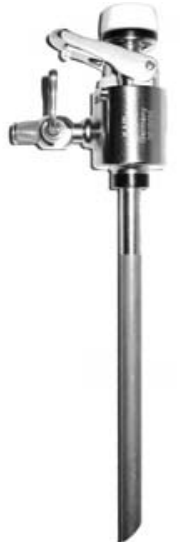

Karl Storz 5

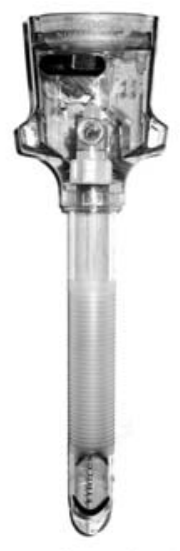

Endopath 12

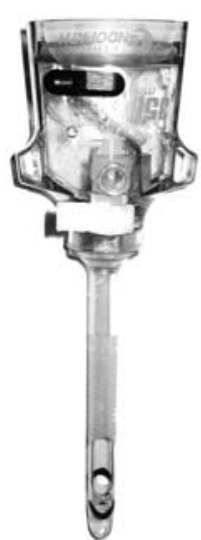

Endopath 5

stick-slip phenomena that arise at the start of a movement or at low velocities may cause large fluctuations in forces. Moreover, movements of the instrument in the trocar may vary quickly, and the direction often reverses (e.g., during tissue dissection). Each reversal in movement direction is accompanied by the same fluctuations in forces that may arise at the start of a movement. Consequently, due to frequent directional changes of movement, force feedback information may be even more distorted at velocities varying around zero than at high velocities. Therefore, the current study particularly investigated whether large fluctuations in frictional forces occur at the beginning of a movement and at low velocities.

We studied the friction characteristics of six trocars commonly used in conventional laparoscopic surgery. The trocars differed both in design and in the materials they comprised. We determined the effect of instrument velocity level and changes in velocity on the amount of friction and on the magnitude of variation. In addition, because some surgeons moisten their instruments with water to minimize friction, we evaluated whether this is indeed an effective method. All measurements were performed in vitro.

\section{Materials and methods}

\section{Trocars}

The main characteristics of the different trocars are summarized in Table 1. Images of the trocars are displayed in Fig. 1. All the trocars were suitable for laparoscopic instruments with a shaft diameter of 5

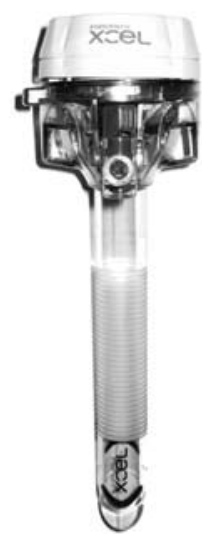

Xcel 12

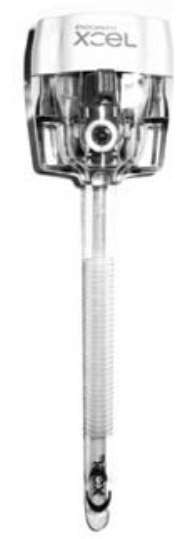

Xcel 5
Fig. 1. Trocars used in the experiment. $\mathrm{mm}$. Some were used in combination with their appropriate reducer caps. The sealing cap of one trocar, the Xcel 12 (Ethicon ENDOSurgery Inc, Cincinnati, Ohio) consisted of multilayered flaps. These had been lubricated already by the manufacturer to reduce friction.

\section{Apparatus}

All measurements were performed with the shaft of a standard laparoscopic grasper (CLICKline; Karl Storz, Tuttlingen, Germany). The shaft had a diameter of $5 \mathrm{~mm}$ and consisted of a stainless steel outer tube insulated with a plastic sheath. It was attached to a slider that allowed translational movement of the shaft through the trocar, which was fixed to a table (Fig. 2). A load cell (FUTEK Advanced Sensor Technology Inc, Irvine, California) was placed in-line with the shaft and slider to measure the forces required to move the shaft through the trocars. The sensor measured the forces at a sample frequency of 1,000 $\mathrm{Hz}$. The slider was moved with the use of a computer-controlled servomotor (Maxon Motor Ag, Sachseln, Switzerland).

A single measurement trial consisted of four inward and four outward movements $35 \mathrm{~mm}$ in amplitude. Each movement contained a phase in which the instrument moved at constant velocity. The instrument was smoothly decelerated and accelerated at movement reversals (Fig. 3). The constant velocity level was varied in the 70 measurement trials (range, $1,2,3 \ldots 70 \mathrm{~mm} / \mathrm{s}$ ). These 70 measurements were performed for each trocar and each condition (dry shaft and moistened shaft). For the moistened shaft condition, a few drops of water were put on the shaft near the entry of the trocar at the start of a measurement trial.

\section{Data analysis}

It was assumed that the forces associated with the inertia of the shaft were negligible, so these were not taken into account. Thus, in the analysis, the output of the load cell was taken as a measure of the friction between the moving shaft and the sealing mechanism of the trocar. 

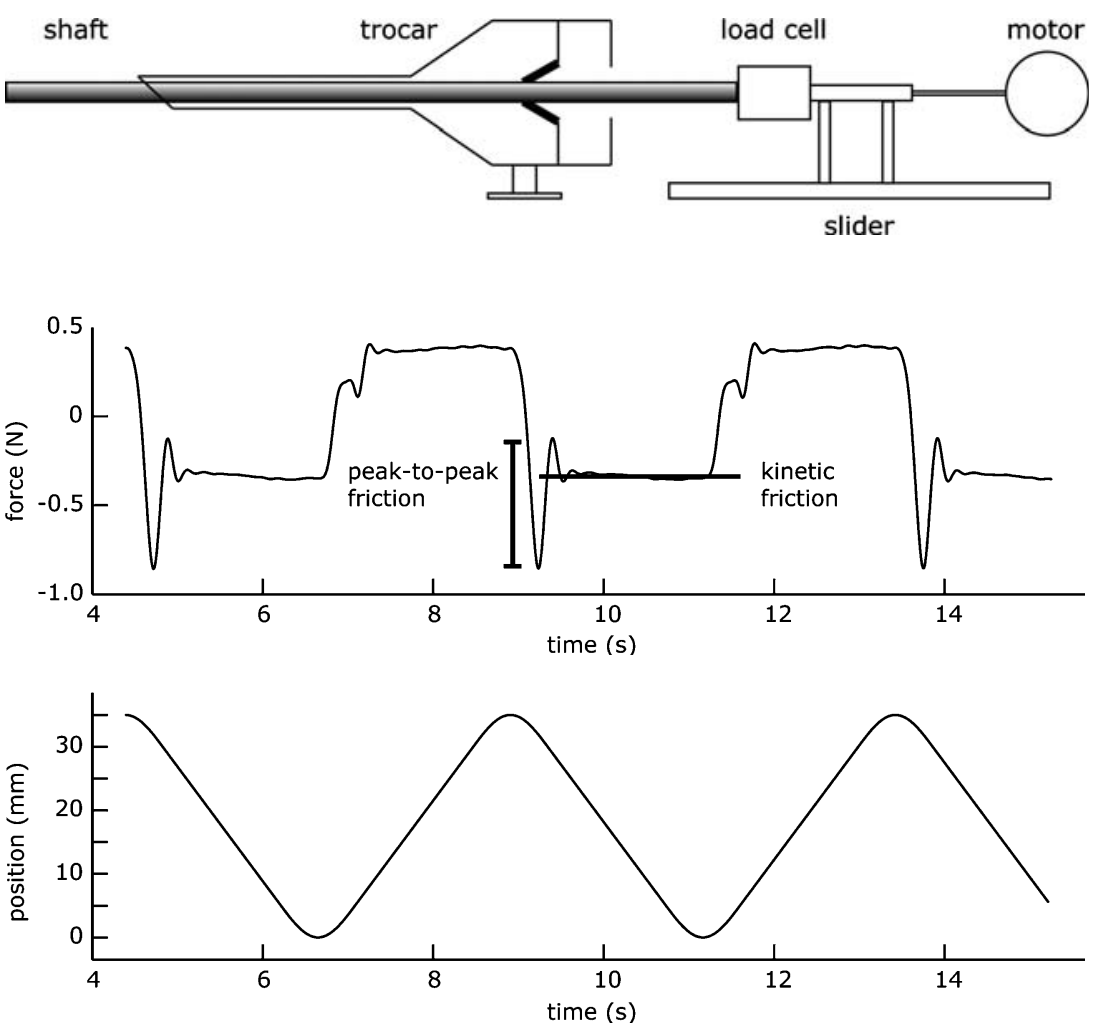

Fig. 2. Schematic drawing of the experimental setup.

Fig. 3. Typical example of a measurement trial. The top graph displays the force measured by the load cell over time. The thick horizontal bar represents the median force measured when the shaft moved at a constant velocity (termed "kinetic friction"). The thick vertical bar represents the peak-to-peak amplitude of the force found during a single movement (termed "peakto-peak friction"). The bottom graph displays the position of the shaft over time.

To characterize the friction in the trocar, two different analyses were used, as explained in Fig. 3. In the first analysis, the magnitude of friction was determined when the shaft moved at a constant velocity (kinetic friction). Kinetic friction represents the friction during steady sliding at a constant velocity. This measure was calculated as the median of the forces during the constant velocity phase for each single movement in a measurement trial. The results for the inward and outward movements were averaged separately per measurement trial to get a single estimate of the kinetic friction for both movement directions and each of the 70 constant movement velocities.

The second analysis was performed to characterize the fluctuations in force attributable to stick-slip (e.g., stretching and slipping of the silicon and rubber parts of the sealing mechanism). Slip and stick are associated with distinct peaks in the force profile. We therefore determined for each movement the peak-to-peak amplitude of the forces present at the beginning of the movement until steady sliding occurred (peak-to-peak friction). Again, the results were averaged over movement direction for each of the 70 movement velocities. Note, however, that the moment of slip, and therefore the occurrence of peak forces, may take place during both the acceleration and constant velocity phases of a movement. Thus, the peaks can be related to forces generated at movement velocities lower than those during the constant velocity phase of a movement.

To evaluate whether moistening of the shaft reduces friction, we calculated the ratios between the friction values obtained for a trocar in the dry and moistened conditions for each movement velocity and direction $(n=140)$. For each trocar, separate one-tailed $t$-tests were performed to determine whether the averaged ratio of kinetic friction or peak-to-peak friction was lower than 1. A $p$ value less than 0.05 was considered significant.

\section{Results}

Figure 3 shows a typical example of a measurement trial. There were clear differences in the friction dynamics between the trocars investigated in this study. Figure 4 illustrates the differences in force profiles for consecutive inward and outward movements with a low (left column) constant velocity phase and a high (right column) constant velocity phase. At very low velocities, stick-slip peaks were present in two of the six trocars and at higher velocities in five of the six trocars.

Both the level of kinetic friction and the level of peak-to-peak friction varied extensively between the trocars. The results for the condition in which the shaft was dry are plotted in Fig. 5. The magnitude of kinetic friction varied between 0.25 and $3 \mathrm{~N}$ for the different trocars and depended on both movement velocity and movement direction. Kinetic friction is greater for higher velocities and outward movements (negative velocity values). The kinetic friction was especially high for the B Braun 5.5 trocar (Melsungen AG, Tuttlingen, Germany) the Karl Storz 5 trocar (Tuttlingen, Germany), and the Endopath 12 trocar (Ethicon ENDOSurgery Inc, Cincinnati, Ohio).

Peak-to-peak friction generally decreased with increasing velocities and was highest for outward movements. The highest peak-to-peak friction attributable to stick-slip was found for the Endopath 12 trocar. For movements with a constant velocity phase lower than $5 \mathrm{~mm} / \mathrm{s}$, stick-slip occurred only with the B Braun 5.5 and the Endopath 12 trocar. For the Karl Storz 5 trocar, no clear stick-slip was present for all velocities. For both Xcel trocars, stick-slip phenomena were present only for intermediate velocities.

Moistening of the shaft reduced the kinetic friction with all trocars. Table 2 reports the means and standard deviations for the ratios between the friction values obtained in the dry and moistened conditions for each trocar. One-tailed $t$-tests showed that for the kinetic friction, all averaged ratios were significantly lower than 1. We could not determine the ratio of peak-to-peak 


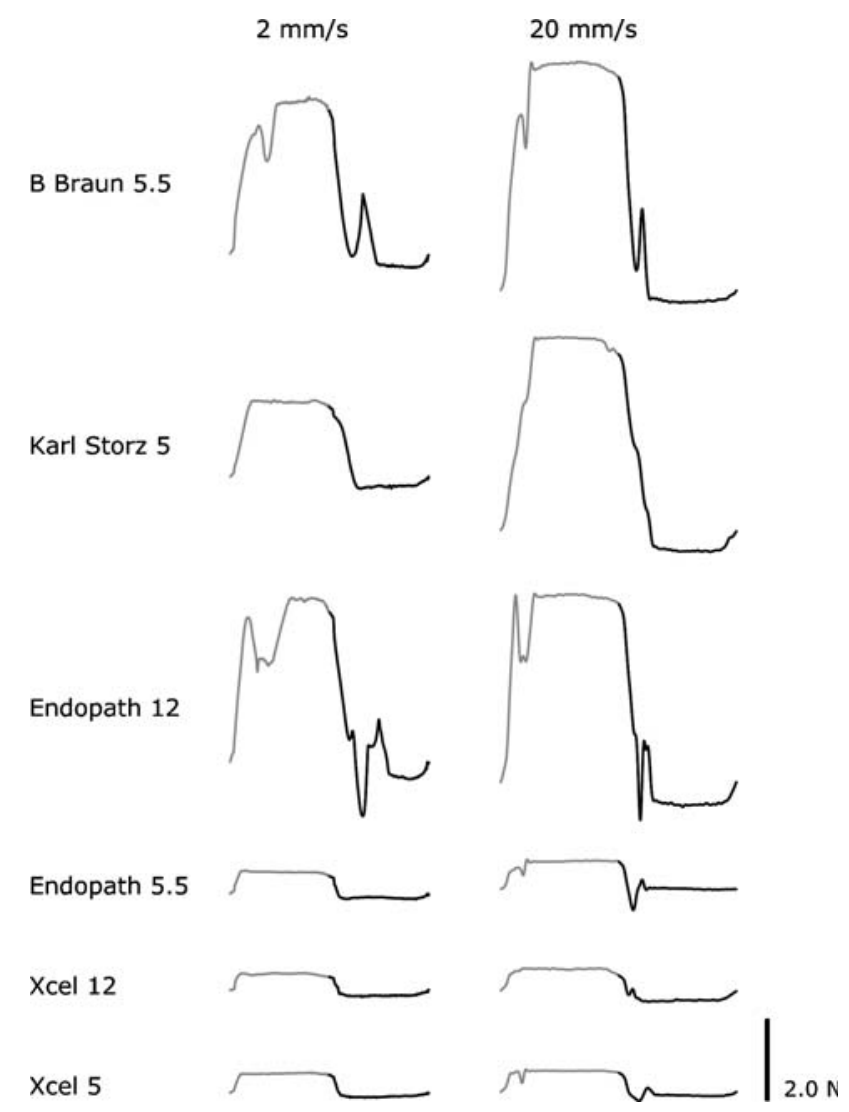

Fig. 4. Typical force profiles for all the trocars. The left column displays the force profiles for the movements with a constant velocity phase of $2 \mathrm{~mm} / \mathrm{s}$. The right column shows the force profiles for the movements with a constant velocity phase of $20 \mathrm{~mm} / \mathrm{s}$. The magnitude of forces $(\mathrm{N})$ is indicated by the thick vertical line at the bottom right of the figure. The gray lines represent the forces during inward movements, and the black lines represent the forces during outward movements.

friction for all trocars. After administering the water, stick-slip was present with only three of the six trocars, and only for a limited number of measurement trials. A very small significant reduction in peak-to-peak friction was found only for the Endopath 5 trocar. After moistening, no stick-slip peaks were found for the Karl Storz 5 or the two Xcel trocars.

\section{Discussion}

This study shows that both the magnitude and the dynamics of friction between laparoscopic instruments and trocars differ extensively across various trocar designs. We further found that the magnitude of friction can be reduced easily by moistening the shaft of the instrument with a few drops of water. Less friction results in less drag of the instrument in the trocar and eases surgical performance. We therefore recommend the use of water or other lubricants in clinical practice.

The level of friction depends on the adhesion and the size of the contact area between the sealing mechanisms of the trocars and the surface of the surgical instrument. Considering that most parts of the trocar sealing mechanisms consist of silicon or rubber, we can easily point out some of the main determinants of high friction levels with trocars. Silicon and rubber typically display strong adhesion. With no movement, the flexible material deforms, following the profile of the instrument, thereby creating a large contact area [9]. The size of the contact area also is influenced by the amount of contact pressure. Therefore, high friction occurs mostly when the sealing cap of the trocar is relatively thick (i.e., creating a large contact area) or has a relatively narrow inner diameter (i.e., inducing high contact pressure). Sealing caps with these properties are common in nondisposable trocars, and were used with the two nondisposable trocars included in this study. Added reducer caps, as used with the Endopath 12, also are relatively narrow, which results in high friction.

Despite the large differences in the friction dynamics of the trocars, there also are clear commonalities in the force profiles. When the laparoscopic instrument starts to move through the trocar, the contact surfaces stick until the force reaches a critical level: the static friction (i.e., the force to start sliding). Beyond this critical force level, slip occurs, and the friction force is reduced. The exact magnitude of this reduction depends in part on the stiffness of the silicon and rubber components of the sealing mechanisms. When the materials are stiff, less deformation occurs. This results in a smaller contact area, and therefore a lower static friction. When the difference between static friction and kinetic friction is small, the reduction in friction during slip also is small. Thus, to prevent large fluctuations in forces at the start of a movement and at movement reversals, the materials used should be stiff. In this situation, however, it is very important that the sealing fits exactly. Too tight a fit will result in high contact pressure and increase both static and kinetic friction. Too loose a fit will result in gas leakage.

To reduce friction, lubricants that create a thin film between the sliding surfaces can be used. In this study, the use of water as a lubricant reduced kinetic friction $15 \%$ to $45 \%$. This means that for trocars with high friction, moistening of the shaft may lead to a reduction in force as great as $1.5 \mathrm{~N}$. We should note that for one trocar, the Xcel 12, parts of the outer seal were lubricated by the manufacturer. The main purpose of this lubricant was to ensure a proper sliding between the partly overlapping flaps of the seal. However, when the instrument moves in the trocar, the lubricant spreads to the contact area between the flaps and the surface of the instrument's shaft. We therefore assume that in this study, the lubricant also affected the friction between the flaps and the shaft of the instrument. The presence of the lubricant may partly explain the relatively low kinetic friction found for this trocar. Still, moistening of the shaft reduced the kinetic friction $20 \%$, showing that even with this trocar, there were beneficial effects of an added lubricant.

The effect of moistening the shaft on the peak-topeak friction was less clear. With some trocars, stickslip disappeared completely. However, with the trocars (or measurement trials) for which stick-slip was still present, there was no reduction in peak-to-peak friction. This probably means that in the latter, the water often is 

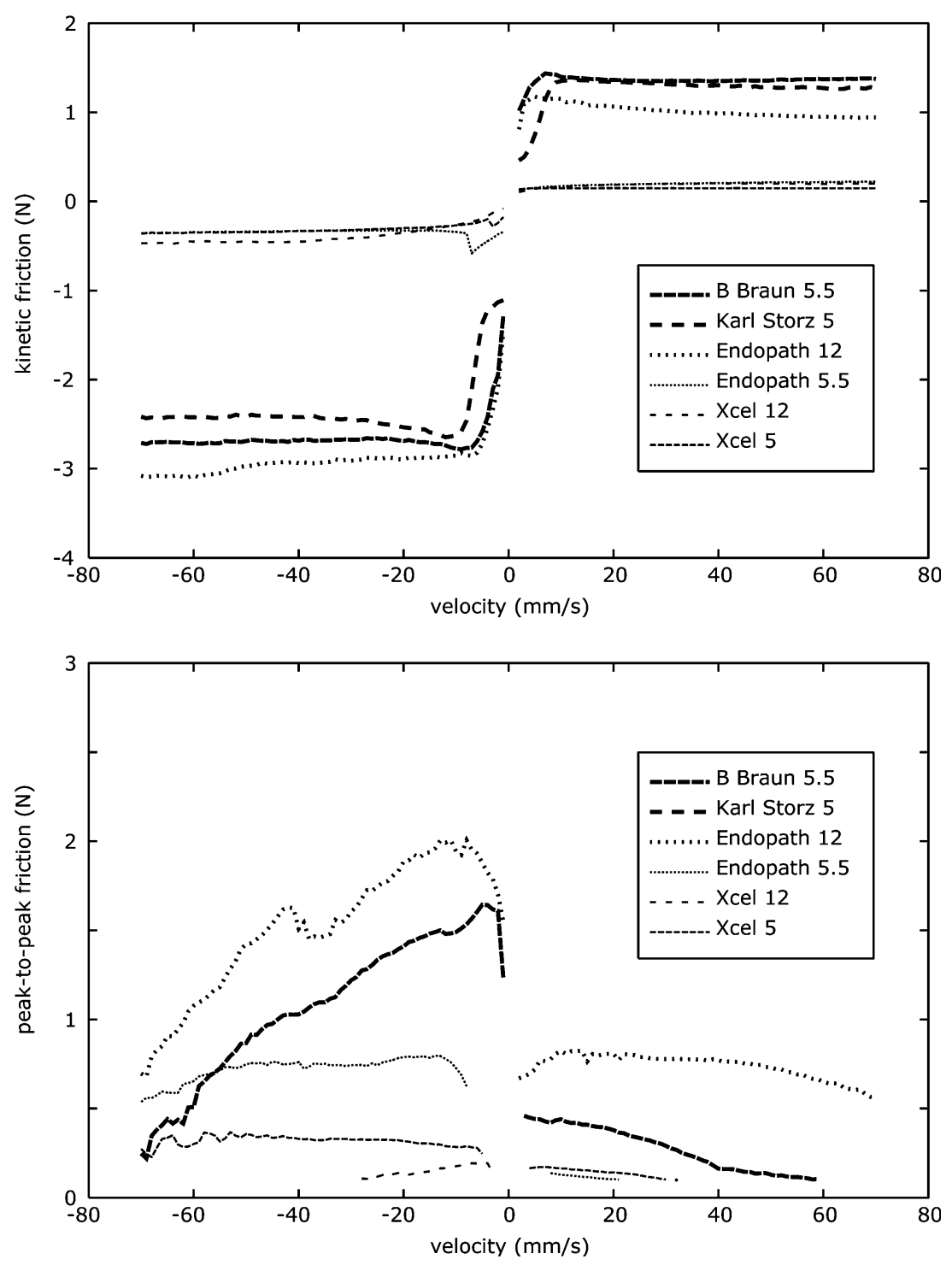

Fig. 5. Friction forces between the trocar and a dry shaft. The top graph shows the kinetic friction found for each trocar for the different velocities. The bottom graph shows the peak-to-peak friction relative to the movement velocity. Negative velocities represent outward movements, and positive velocities represent inward movements. Note that the peak-to-peak friction could not be determined for some trocars and velocities because there was no stick-slip.

Table 2. Kinetic and peak-to peak friction ratios between the dry and moistened conditions

\begin{tabular}{|c|c|c|c|c|c|c|c|c|}
\hline \multirow[b]{2}{*}{ Trocar } & \multicolumn{4}{|c|}{ Kinetic friction ratio $^{\mathrm{a}}$} & \multicolumn{4}{|c|}{ Peak-to-peak friction ratio ${ }^{b}$} \\
\hline & Mean & SD & $n$ & $p$ Value & Mean & SD & $n$ & $p$ Value \\
\hline B Braun 5.5 & 0.56 & 0.07 & 140 & $<0.001$ & 1.04 & 0.51 & 101 & NS \\
\hline Karl Storz 5 & 0.71 & 0.12 & 140 & $<0.001$ & - & - & - & - \\
\hline Endopath 12 & 0.74 & 0.14 & 140 & $<0.001$ & 1.00 & 0.18 & 137 & NS \\
\hline Endopath 5.5 & 0.85 & 0.09 & 140 & $<0.001$ & 0.99 & 0.06 & 74 & 0.019 \\
\hline Xcel 12 & 0.80 & 0.25 & 140 & $<0.001$ & - & - & - & - \\
\hline Xcel 5 & 0.54 & 0.11 & 140 & $<0.001$ & - & - & - & - \\
\hline
\end{tabular}

$\mathrm{SD}$, standard deviation; NS, not significant

a One kinetic friction ratio was calculated for each measurement trail (i.e., each velocity and direction; $n=140$ ) and each trocar. All averaged kinetic friction ratios are significantly below 1.0

${ }^{\mathrm{b}}$ Peak-to-peak friction ratios could be calculated only when stick-slip peaks were present for a certain velocity and direction in both conditions

squeezed out of the contact area. At low velocities, the deforming materials of the sealing push the water away. Only during steady sliding is a film of water formed between the sealing and the shaft so that the kinetic friction is reduced but the peak-to-peak-friction remains the same. To reduce the peak-to-peak friction, other lubricants more viscous than water may be more beneficial. This, however, requires further study.

We found that at high instrument velocities, the kinetic friction generated in trocars can be as great as the forces associated with instrument-tissue interaction. This result is in line with the findings of Picod et al. [10, 
11]. Our study further shows that even when instrument velocity is low, force feedback on the instrument's interactions with the surgical environment can be distorted by dynamic changes in friction between the instrument and the sealing mechanisms of the trocars. The dynamic changes in friction arise each time an instrument starts to move or movement direction reverses. Tissue manipulation, among other high-precision tasks, typically involves low instrument velocities and many changes in movement direction. Our results suggest that the properties of some currently used trocars may be disadvantageous for dexterous tissue manipulation. Although it is unknown how a reduced quality of force feedback affects appropriate force application [8], large fluctuations in friction will disrupt smooth instrument motion and may therefore complicate surgical performance.

\section{Conclusions}

At low instrument velocities, dynamic changes in friction occur in trocars. This distorts force feedback and motion control in high-precision tasks such as tissue manipulation. Friction magnitude can be reduced easily by changing the design of trocar sealing mechanisms or by using lubricants.

Acknowledgments. We thank Karl Storz Endoscopie Nederland BV, Ethicon Endo Surgery (Johnson \& Johnson Medical BV), and Ae- sculap (B. Braun Medical BV) for providing the trocars used in this study.

\section{References}

1. De Visser H, Heijnsdijk EA, Herder JL, Pistecky PV (2002) Forces and displacements in colon surgery. Surg Endosc 16: 1426-1430

2. Den Boer KT, Herder JL, Sjoerdsma W, Meijer DW, Gouma DJ, Stassen HG (1999) Sensitivity of laparoscopic dissectors: what can you feel? Surg Endosc 13: 869-873

3. Dubois P, Thommen Q, Jambon AC (2002) In vivo measurement of surgical gestures. IEEE Trans Biomed Eng 49: 49-54

4. Elmer FJ (1997) Nonlinear dynamics of dry friction. J Phys A Math Gen 30: 6057-6063

5. Hager-Ross C, Johansson RS (1996) Nondigital afferent input in reactive control of fingertip forces during precision grip. Exp Brain Res 110: 131-141

6. Heijnsdijk EA, van der Voort M, de Visser H, Dankelman J, Gouma DJ (2003) Inter- and intraindividual variabilities of perforation forces of human and pig bowel tissue. Surg Endosc 17: 1923-1926

7. Johansson RS, Westling G (1987) Signals in tactile afferents from the fingers eliciting adaptive motor responses during precision grip. Exp Brain Res 66: 141-154

8. Lamata P, Gomez EJ, Sanchez-Margallo FM, Lamata F, Antolin M, Rodriguez S, Oltra A, Uson J (2006) Study of laparoscopic forces perception for defining simulation fidelity. Stud Health Technol Inform 119: 288-292

9. Persson BNJ (1998) Sliding friction physical principles and applications. Springer, Berlin

10. Picod G, Jambon AC, Dubois P, Vinatier D (2003) Measurement of the friction in the sheath of the trocars used in laparoscopy. ITBM-RBM Revue Européenne de Technologie Biomédicale 24: $217-222$

11. Picod G, Jambon AC, Vinatier D, Dubois P (2005) What can the operator actually feel when performing a laparoscopy? Surg Endosc 19: 95-100 